\title{
UAV PHOTOGRAMMETRY AND VHR SATELLITE IMAGERY FOR EMERGENCY MAPPING. THE OCTOBER 2020 FLOOD IN LIMONE PIEMONTE (ITALY)
}

\author{
L. Teppati Losè̀ ${ }^{*}$, F. Chiabrando ${ }^{1}$, F. Giulio Tonolo ${ }^{1}$, A. Lingua ${ }^{2}$ \\ ${ }^{1}$ LabG4CH, Department of Architecture and Design (DAD) - Politecnico di Torino, Viale Mattioli 39, 10125 Torino (Italy) \\ (lorenzo.teppati, filiberto.chiabrando, fabio.giuliotonolo)@polito.it \\ ${ }^{2}$ Laboratory of Photogrammetry, Geomatics \& GIS, Department of Environment, Land and Infrastructures Engineering (DIATI) - \\ Politecnico di Torino, Corso Duca degli Abruzzi 24, 10129 Torino (Italy), (andrea.lingua@polito.it)
}

\section{Commission III, ICWG III/IVa}

KEY WORDS: UAVs, Direct Georeferencing, Emergency Mapping, VHR, Satellite, SfM.

\begin{abstract}
:
Heavy rain between the 2nd and 3rd of October 2020 severely affected the area of Limone Piemonte, Piemonte Region (Italy). The consequence of those two days of rain was a flood that, starting from the hamlet of Limonetto severely damaged the areas close to the riverbed of the Vermegnana river and the related hydrographyc network. A synergistic multi-sensor and multi-scale approach for documenting the affected areas using VHR satellite images and UAVs (Uncrewed Aerial Vehicles) is presented. The pro and cons in terms of level of detail and processing strategies are reviewed with a focus on the workflows adopted for processing large UAV datasets. A thorough analysis of the 3D positional accuracy achievable with different georeferentation strategies for UAVs data processing is carried out, confirming that if an RTK (Reale Time Kinematic)-enabled GNSS (Global Navigation Satellite System) receiver is available on the UAV platform and proper acquisition guidelines are followed, the use of GCPs (Ground Control Points) is not impacting significantly on the overall positional accuracy. Satellite data processing is also presented, confirming the suitability for large scale mapping.
\end{abstract}

\section{INTRODUCTION}

In the last decades, the increased incidence of catastrophic events has posed new obstacles to the operators involved in preparedeness, response, recovery, and mitigation phases of the emergency management cycle. In this context, emergency mapping based on both satellite has been used as primary source of information in the last decade, being generally available shortly after the event (Boccardo \& Giulio Tonolo, 2015; Tralli et al., 2005). Nevertheless, the level of detail offered by satellite imagery $(\mathrm{max} G S D=0.3 \mathrm{~m}$ ) and the off-nadir angle constraints may be limiting when assessing damages. In the last years, new solutions have been developed thanks to the broader availability of UAVs, as reported in (Calantropio et al., 2018; Chiabrando et al., 2019; Duarte et al., 2017). Nevertheless, the integration of these platforms in operational services is not yet consolidated.

\subsection{The Limone Piemonte case study}

The case study presented in this research is focused on the area of Limone Piemonte, a village located in the southwest of Piemonte region, Italy that was hit by a major flood induced by heavy precipitations between the $2^{\text {nd }}$ and $3^{\text {rd }}$ of October 2020 (Figure 1). The hydric systems and the riverbed of the Vermegnana river were severely hit by these events.
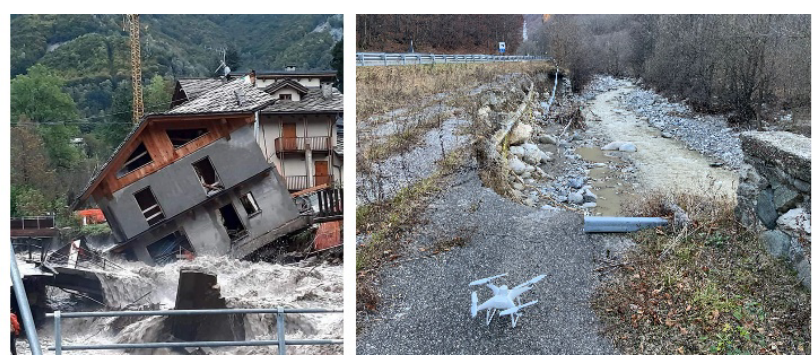

Figure 1. Some of the damages of October 2020 flood in the area of Limone Piemonte, Piemonte region, Italy.

A post-event very high resolution (VHR) satellite imagery was acquired and processed, mainly to identify the most affected areas. Moreover in order to follow a multi-sensor, multiplatform, and multi-scale integrated approach, an acquisition campaign over case study area was planned, focusing on UAVs acquisitions for damage assessment purposes.

The activities in the area of Limone Piemonte were jointly organised and carried out by the Laboratory of Geomatics for Cultural Heritage (Department of Architecture and Design), the Laboratory of Photogrammetry, Geomatics \& GIS (Department of Environment, Land and Infrastructures Engineering) and the student team DiRECT (Disaster and Recovery Team, https://www.g4ch.polito.it/wordpress/team-direct/) of the Politecnico di Torino.

\footnotetext{
Corresponding author
} 


\section{MATERIALS AND METHODS}

A post-event Very High Resolution (VHR) satellite image was acquired and processed. According to the technical specifications of the GeoEye-1 platform $(\mathrm{GSD}=0.46 \mathrm{~m}$, panchromatic and GSD $=1.84$ multispectral) the expected nominal map scale of the derived orthoimagery is 1:5000, enabling a post-disaster damage assessment: in this research activity satellite data is used for data integration and comparison. On the other hand in order to acquire data at a larger map scale a UAV survey campaign has been carried out with the main goal of mapping a $100 \mathrm{~m}$ buffer zone around the Vermegnana riverbed as well as the urban area of Limone Piemonte to derive metric dataset supporting the damage assessment in the recovery and reconstruction phase. The campaign for data acquisition was performed on $24^{\text {th }}$ and $25^{\text {th }}$ November 2020. The activity was designed to deliver metric added-value products with a minimum nominal map scale of 1:500, with a positional precision lower than $0.1 \mathrm{~m}$ and tolerance lower than $0.2 \mathrm{~m}$ (according to common mapping standards in Italy).

\subsection{Fieldwork and data acquisition}

The documentation of the area of interest in Limone Piemonte was carefully designed to respond to different needs: during the fieldwork, it was necessary to map the riverbed and the damages in the urban area of Limone and along the Strada Statale 20 (the State Highway that was partially damaged by the flood). The area covered by the UAV surveys is $\sim 1.9 \mathrm{~km}^{2}(\sim 1.5$ $\mathrm{km}^{2}$ covering the Limone Piemonte built-up area) and the flight planning was carefully designed to cover all the territory interested by the survey. The research presented in this manuscript will focus only on the area of Limone Piemonte village: the related flight area was divided into seven different parts (as shown in Figure 2) to optimize the battery usage, the flight time, and the parallel field activities. A DJI Phantom 4 $\mathrm{RTK}^{1}$ multi-rotor UAV platform (1" CMOS 20 MP sensor, equipped with multi-frequency and multi-constellation GNSS receiver with RTK capability) was used during the surveys. For each of the seven areas, three different flights were carried out ( Table 1) for a total number of 21 flights that generated 5225 images.

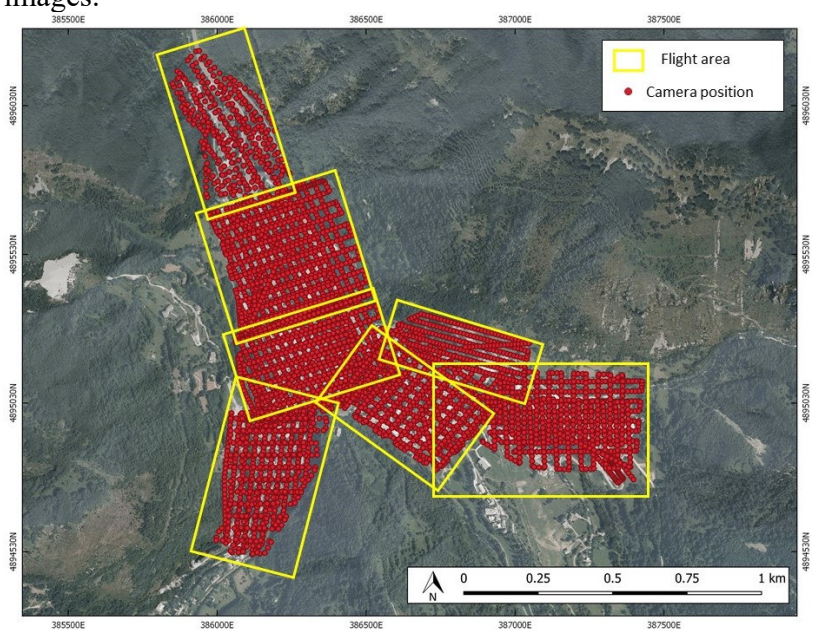

Figure 2. UAV flights performed in the area of Limone Piemonte village. 7 flights were carried out (yellow rectangles) and a total number of 5225 images were acquired (red dots)

${ }^{1}$ A complete list of this system's specifications can be found at: https://www.dji.com/it/phantom-4-rtk/info\#specs

\begin{tabular}{|c|l|c|c|}
\hline ID & Description & Flight lines & $\begin{array}{c}\text { Camera } \\
\text { Orientation }\end{array}$ \\
\hline 1 & Nadiral & $\sim$ North-South & Nadiral \\
\hline 2 & Oblique 1 & $\sim$ North-South & $45^{\circ}$ \\
\hline 3 & Oblique 2 & $\sim$ Est-West & $45^{\circ}$ \\
\hline
\end{tabular}

Table 1. UAVs flights' main characteristics for each of the 7 areas documented.

The average flight altitude was $\sim 80 \mathrm{~m}$ height above the take-off point, the overlap between images was set to $80 \%$ frontal and $75 \%$ side and the average expected GSD (Ground Sampling Distance) was $2.7 \mathrm{~cm} /$ pixel. All the flights used an NRTK (Network Real-Time Kinematic) solution for UAV positioning and camera Direct Georeferencing (DG), thanks to the corrections sent via a GNSS Networked Transport of RTCM via Internet Protocol (NTRIP). As reported in previous research (Peppa et al., 2019; Štroner et al., 2021; Taddia et al., 2020; Teppati Losè et al., 2020a, 2020b) an NRTK-based DG solution enables centimeter-level accuracy in camera positioning and thus in the overall photogrammetric processing. Nevertheless, at least two different conditions should be met to adopt this approach: the presence of a network of CORSs (Continuously Operating Reference Stations) covering the area (including a subscription if required) and the presence of a stable Internet connection. Both these conditions were met in Limone Piemonte, enabling a DG approach. The research of the authors previously reported underlined the cost-effective benefits of using this platform for a DG approach, as well as some issues that needed further attention and analyses. Bearing this in mind and to allow a rigorous validation and investigation of the proposed approach a set of points used for the satellite image orthocorrection and in the photogrammetric process as GCPs (Ground Control Points) and or as CPs (Check Points) was set and measured by mean of traditional topographic techniques with an accuracy of few centimeters (Figure 3).

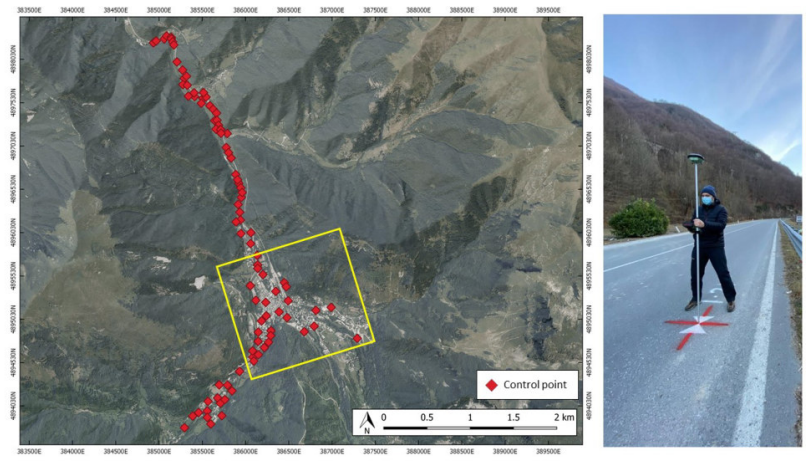

Figure 3. The position of the surveyed points materialized in all the area of interest (a portion of the State Highway number 20 and the urban area of Limone Piemonte).

The surveyed points were materialised following different methods: i) $40 \times 40 \mathrm{~cm}$ coded plastic target fixed on the ground by means of metallic nails (

Figure 4, right), ii) target traced on the terrain using spray paint of different colours (Figure 3, right), ii) natural features (Figure 5 , right). Each control point was then measured adopting a NRTK approach using a GNSS receiver connected with the network of CORSs.

In the overall area object of the survey 99 points were materialized and measure (Figure 3, left), among which 22 were placed in the area of Limone Piemonte and were used for the tests reported in this manuscript (Figure 4). 

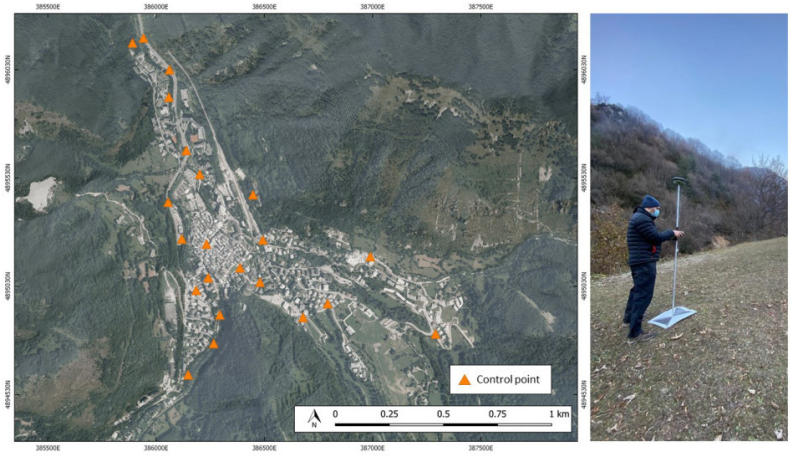

Figure 4. Distribution of surveyed points (left) materialized and measured (right) in the area of Limone Piemonte village.

\subsection{Satellite data}

A preliminary analysis of available VHR satellite imagery in the relevant archives of satellite data providers was carried out, using as search criteria the spatial resolution (nominal GSD $\leq$ $0.5 \mathrm{~m}$ ), the acquisition date (as close as possible to the event date), and the cloud coverage (enabling the visual interpretation on the area of interest). A subset (covering the overall area of interest shown in Figure 3) of a GeoEye-1 image acquired on October 5th, 2020 at 10:27 UTC (3 days after the flood event) has been purchased. The image was acquired with a mean offnadir angle of $23.3^{\circ}$, resulting in a mean GSD of $0.48 \mathrm{~m}$.

The image was delivered as level 2A (radiometrically corrected) standard pansharpened data, including 4 bands ( 3 visible and 1 Near Infrared) resampled at the spatial resolution of $0.5 \mathrm{~m}$.

\section{DATA PROCESSING}

The processing of the satellite image was performed following a standard workflow (section 3.1). On the other hand the processing of a UAV dataset with so many images posed a series of issues. Two approaches were thus tested in order to define the better strategy to optimize this phase: i) a processing of the seven image blocks in three separate blocks (Strategy 1) and ii) a processing of all the blocks in a single solution (Strategy 2). Several aspects have been evaluated: the number and distribution of GCPs and CPs in each of the processing block, how to deal with the overlapping area between adjacent blocks, how to handle the integration between the different products derived (mainly orthoimages and DSM), the radiometric quality of the final orthoimage, etc.

\subsection{Satellite data processing}

The GeoEye-1 satellite image has been processed through a standard orthocorrection approach using an RPC (Rational Polynomial Coefficients) model with a $1^{\text {st }}$ order correction through the OrthoEngine module Geomatica BANFF suite.

5 GCP have been manually identified on the image, using as reference data:

- As is reported before natural markers that have been measured with centimetric accuracy using an RTK GNSS receiver during the UAV surveys campaign (Figure 5 and section 2.1)

- homologous point identified on UAV orthoimagery with centimetric accuracy extracting the height form the related DSM
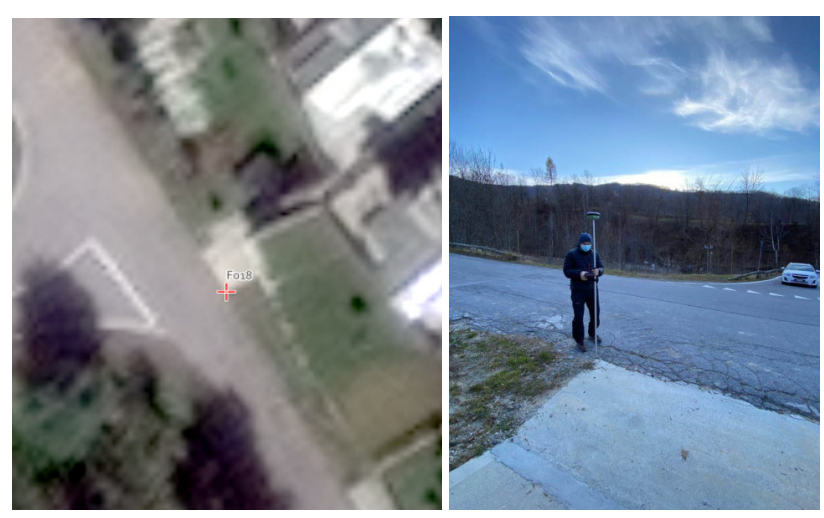

Figure 5. Example of GCP used for satellite image geometric correction. Visual identification on the satellite image (left, red cross) and RTK GNSS measurement in the field (right).

The residuals (in terms of RMSe, Root Mean Square error) on 5 GCPs, using an RPC model with a $1^{\text {st }}$ order correction, are (as expected) lower of the mean GSD, specifically: $\mathrm{RMSeX}=0.43$ $\mathrm{m}, \mathrm{RMSeY}=0.39 \mathrm{~m}, \mathrm{RMSeZ}=0.25 \mathrm{~m}$

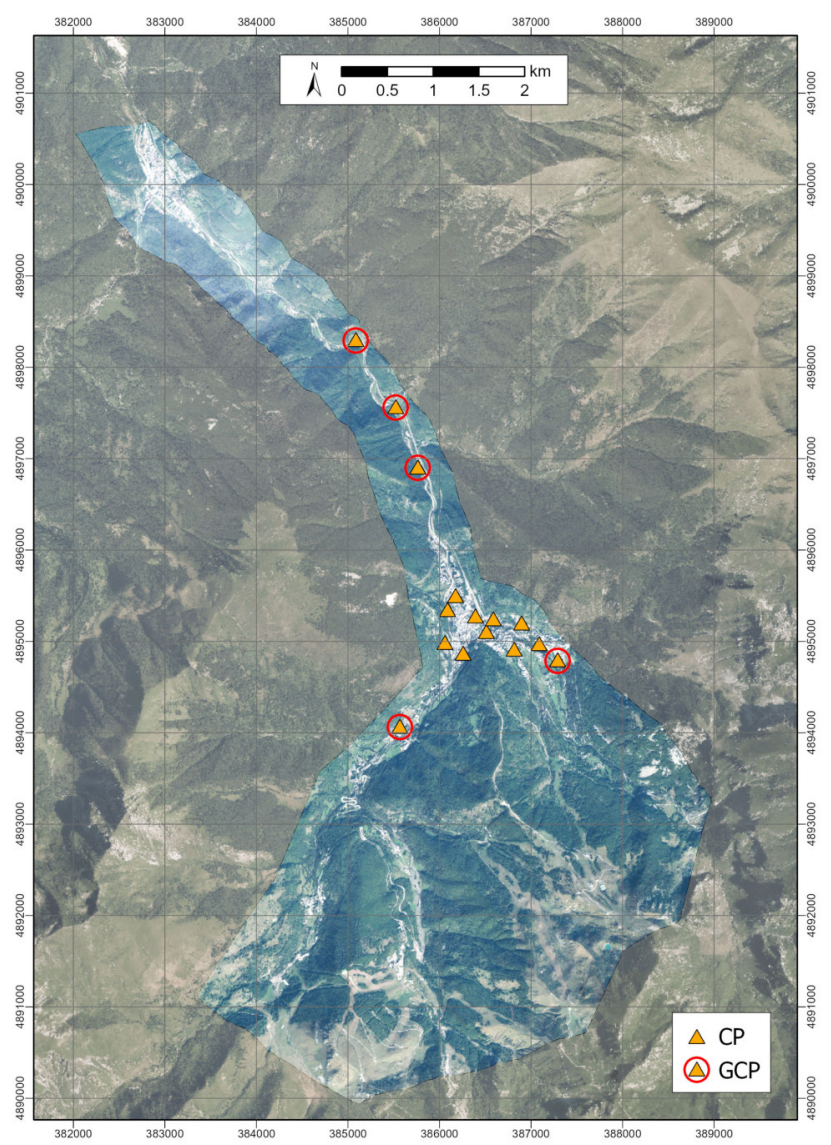

Figure 6. Spatial distribution of CP and GCP overlapped to the satellite orthoimagery.

The image has been ortocorrected using as ancillary data the DTM provided by the Regione Piemonte regional mapping agency, characterised by a vertical accuracy in the range from $0.3 \mathrm{~m}$ to $0.6 \mathrm{~m}$ (being derived from aerial LiDAR surveys) and an horizontal resolution of $5 \mathrm{~m}$. According to the operational experience of the authors and as confirmed in (Aguilar et al., 2012), a limited number of GCP is enough to achieve an optimal geometric correction of the bias induced by the vendor- 
provider RPC coefficients. This assumption is cross-checked by means of the evaluation of the residuals on 10 CPs (covering only the area of interest of Limone Piemonte), manually identified on the satellite orthoimage using UAS orthoimagery as reference data.

The total RMSxy calculated on the $10 \mathrm{CPs}$ is equal to $0.55 \mathrm{~m}$ (same order of magnitude of the nominal GSD) confirming that the $2 \mathrm{D}$ positional accuracy of the proposed approach is meeting emergency mapping requirements and fit a 1:5000 map scale (at least).

The distribution of GCP and CP overlapped to the generated satellite orthoimage $(\mathrm{GSD}=0.5 \mathrm{~m})$ is shown in Figure 6 .

\subsection{Strategy 1. Processing of three blocks}

The first processing strategy foresaw the combination of the seven image blocks acquired in the field in three different processing blocks. The three blocks were designed to guarantee a sufficient overlap between the different areas and were structured as reported in Figure 7.

The division of the data available for the area of Limone Piemonte, was performed considering also the number of images acquired to achieve a good balance between the images included in each block as is reported in Table 2.

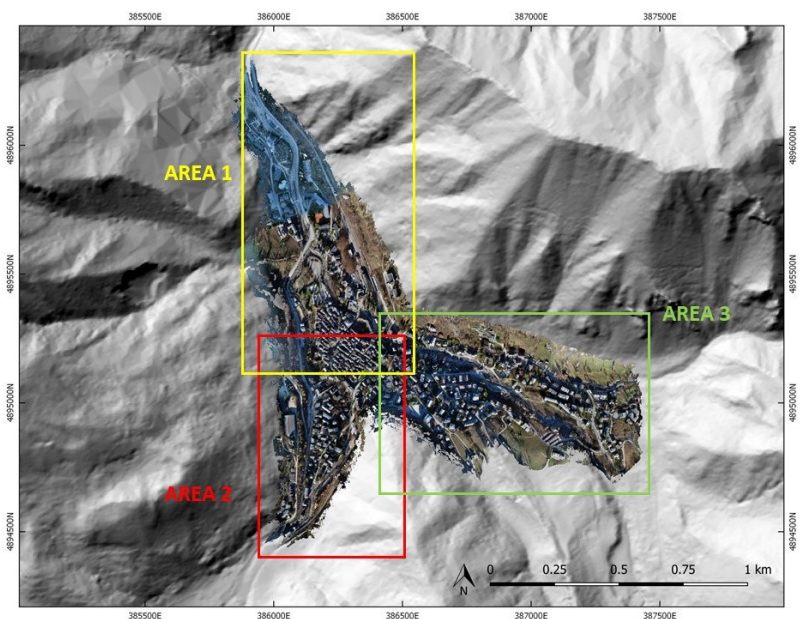

Figure 7. Processing Strategy 1. The 3 areas highlighted yellow, red and green represent the different processing blocks in which images acquired in the field were grouped.

The processing of the three blocks was performed through the commercial software solution Agisoft Metashape (v. 1.7.1) following the consolidated SfM approach (image matching and tie points extraction, Bundle Block Adjustment, point cloud densification and generation of added value metric products such as DSM and orthophoto). For comparison purposes, it was decided to follow a standard photogrammetric pipeline for the first processing strategy, i.e. without using a DG approach and exploiting measured GCPs. The geolocation information embedded in the EXIF (exchangeable image file format) file of the images was erased and tie points extraction, Bundle Block Adjustment and densification without any a-priori information about camera position were exploited. This decision was supported by the idea of further testing the standard photogrammetric workflow with a very large dataset when a UAV with RTK capability is not available. As described in section 4.1, the analysis of the results of this strategy identified different issues that confirmed the opportunity to adopt the second processing strategy (processing in single solution) when possible.

\subsection{Strategy 2. Processing in a single solution}

For the second processing strategy, all the images acquired in the field were processed in a single solution using Agisoft Metashape software. The first aspect that was considered was the size of this dataset in relation to the hardware performances. While the first processing step (the so-called alignment - i.e., tie points extraction and Bundle Block Adjustment) was still manageable with a high-end desktop computer, the subsequent phases of pointcloud densification, DSM generation and orthoimage generation were too demanding in term of computational resources. To solve this issue it is possible to adopt different strategies as for example the Agisoft Cloud computing solution (that was at the time of the processing still offered as an experimental stage) or splitting the processing on different computer creating a local network with one computer working as main server and the others as processing nodes. The cloud processing time is highly influenced by the time needed for data sync between desktop and cloud; the images on the area of Limone Piemonte weight around 43 GigaBytes and their upload on the cloud required quite some time. While the cloud solution doesn't seem to be competitive with respect to the same processing performed on the desktop PC for the images alignment step, it was definitely crucial for the subsequent point cloud densification and product generation.

For the second strategy three different approaches where therefore adopted: a standard approach without measured camera positions and with GCPs (as for Strategy 1 but in a single solution), a standard approach with measured camera position and with GCPs (GeoTag in Table 2) and a DG approach exploiting the RTK capabilities of the Phantom 4 RTK (measured camera position without GCPs).

Particular attention has been devoted to testing different GCPs/CPs configurations during the processing of the dataset with the GeoTag/DG approach. Several tests have been performed, resulting in 4 different configurations ( 1 for the standard approach, 2 for the GeoTag and 1 for the DG approach); these configurations have been identified with a letter and are described in Table 2.

\begin{tabular}{|l|c|c|c|c|}
\hline ID & $\mathrm{N}^{\circ}$ images & Approach & GCPs & CPs \\
\hline \multirow{4}{*}{$\mathrm{A}$} & \multirow{3}{*}{5225} & Standard & 11 & 11 \\
\cline { 1 - 3 } $\mathrm{B}$ & DG & 0 & 22 \\
\cline { 4 - 5 } & & GeoTag & 6 & 16 \\
\cline { 4 - 5 } $\mathrm{C}$ & GeoTag & 11 & 11 \\
\hline $\mathrm{D}$ & &
\end{tabular}

Table 2. Processing strategy 2 , georeferencing approach and GCPs/CPs configuration

As reported by different authors (Agüera-Vega et al., 2017; Bolkas, 2019; Teppati Losè et al., 2020; Tonkin \& Midgley, 2016) the number and distribution of GCPs and CPs can highly impact on the overall positional accuracy of the photogrammetric processing. For this reason, three different GCPs/CPs configurations have been tested during processing Strategy 2 (Figure 8). The distribution of points has been designed considering the overall conformation of the area of Limone Piemonte and ensuring an homogenous distribution of the different types of points (GCPs or CPs). It should be noted that control points selected as GCPs and CPs are the same in configurations $\mathrm{A}$ and $\mathrm{D}$ to grant a coherent comparison. 

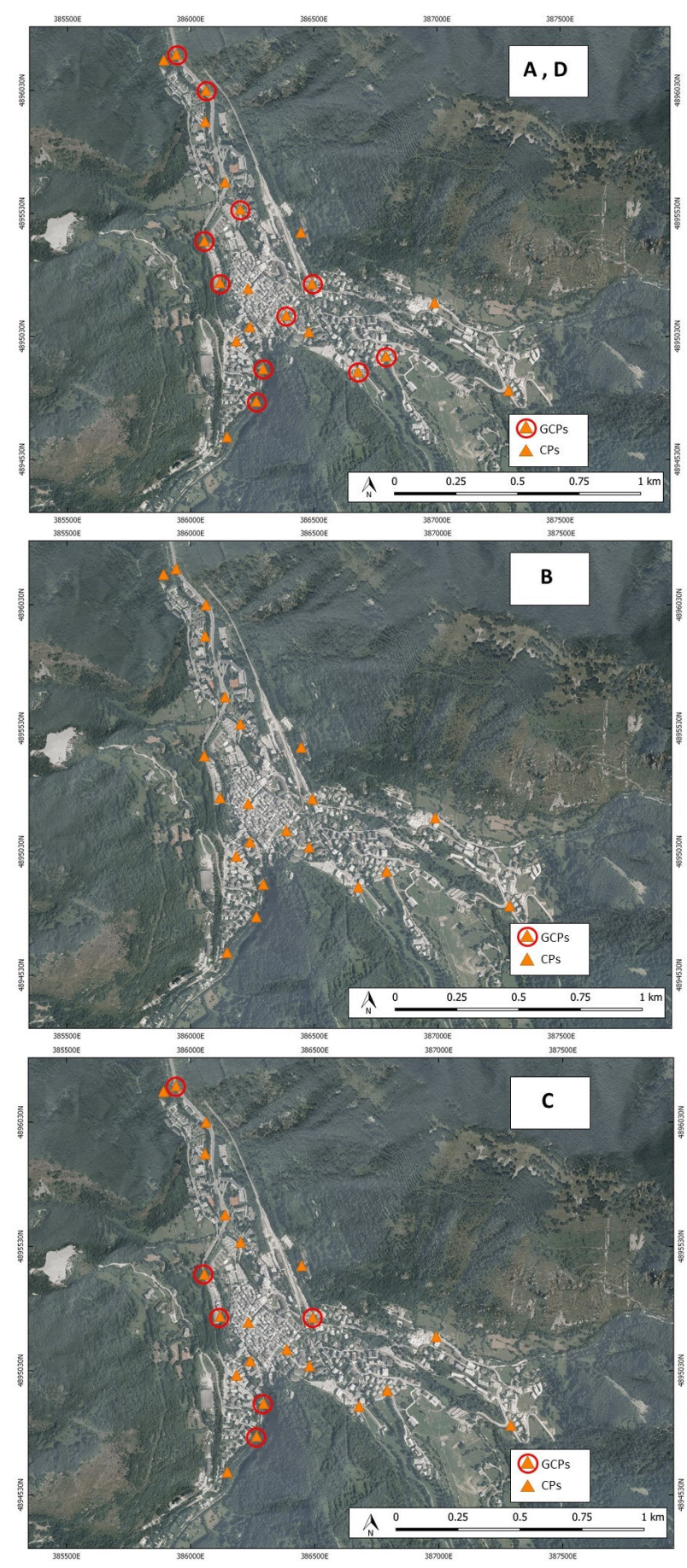

Figure 8. GCPs/CPs configuration for the different processing approaches in a single solution (strategy 2)

\section{DATA ANALYSIS AND RESULTS}

\subsection{Processing strategy}

The two adopted processing strategies were carefully evaluated and analysed from different perspectives. Firstly the positional accuracy of the overall photogrammetric processing was assessed. As far as Strategy 1 is concerned, it should be noted that due to the division of the area into three different processing blocks, the number of control points for each block is limited. The RMSe value on GCPs for each of the areas is within the expected range, as shown in Table 3: however, the low number and the distribution of ground control points was not always sufficient to grant a coherent set up of GCPs/CPs.

\begin{tabular}{|l|c|c|c|}
\hline $\begin{array}{l}\text { Image } \\
\text { block }\end{array}$ & $\mathrm{N}^{\circ}$ images & $\begin{array}{c}\text { RMSe } \\
(\mathrm{GCPs})\end{array}$ & $\begin{array}{c}\mathrm{N} . \text { of } \\
\text { GCPs }\end{array}$ \\
\hline AREA 1 & 1596 & 0.027 & 11 \\
AREA 2 & 1713 & 0.015 & 11 \\
AREA 3 & 1916 & 0.023 & 8 \\
\hline
\end{tabular}

Table 3. Processing Strategy 1, main parameters of the processing of the three blocks.

The large number of GCPs required for Strategy 1 is one of the main reason why Strategy 2 seems optimal when processing a very large dataset. The main aim of the analysis of the results related to Strategy 2 is to evaluate the performances of DG approach in comparison with the traditional workflow with/without camera positions and depending on the adopted GCPs/CPs configuration. The first analysis was performed on the RMSe of GCPs/CPs of the four configurations, as shown in Table 4.

\begin{tabular}{|c|c|c|c|c|c|c|}
\hline & Approach & $\begin{array}{l}\text { GCPs/ } \\
\text { CPs }\end{array}$ & $\begin{array}{l}\text { RMSe } \\
\text { X (m) }\end{array}$ & $\begin{array}{l}\text { RMSe } \\
\text { Y (m) }\end{array}$ & $\begin{array}{l}\text { RMSe } \\
\text { Z (m) }\end{array}$ & $\begin{array}{l}\text { RMSe } \\
\text { TOT } \\
\text { (m) }\end{array}$ \\
\hline \multirow[t]{2}{*}{ A } & \multirow[t]{2}{*}{ Standard } & $\begin{array}{l}11 \\
\text { GCPs }\end{array}$ & 0.011 & 0.011 & 0.014 & 0.021 \\
\hline & & $\begin{array}{l}11 \\
\text { CPs }\end{array}$ & 0.052 & 0.033 & 0.051 & 0.081 \\
\hline \multirow[t]{2}{*}{ B } & \multirow[t]{2}{*}{ DG } & $\begin{array}{l}0 \\
\text { GCPs } \\
\end{array}$ & 1 & 1 & I & 1 \\
\hline & & $\begin{array}{l}22 \\
\mathrm{CPs}\end{array}$ & 0.016 & 0.015 & 0.055 & 0.061 \\
\hline \multirow[t]{2}{*}{$\mathrm{C}$} & \multirow[t]{2}{*}{ Geotag } & $\begin{array}{l}6 \\
\text { GCPs }\end{array}$ & 0.009 & 0.006 & 0.017 & 0.021 \\
\hline & & $\begin{array}{l}16 \\
\text { CPs }\end{array}$ & 0.016 & 0.016 & 0.057 & 0.062 \\
\hline \multirow[t]{2}{*}{$\mathrm{D}$} & \multirow[t]{2}{*}{ Geotag } & $\begin{array}{l}11 \\
\text { GCPs } \\
\end{array}$ & 0.010 & 0.080 & 0.019 & 0.023 \\
\hline & & $\begin{array}{l}11 \\
\text { GCPs } \\
\end{array}$ & 0.018 & 0.019 & 0.053 & 0.059 \\
\hline
\end{tabular}

Table 4. RMSe on control points for different processing configuration: Standard approach with 11 GCPs (A), DG without GCPs (B), GeoTag with 6 GCPs (C), GeoTag with 11 GCPs (D)

From this first analysis, considering that the accuracies of the different configurations always met the requirements for a map at the nominal scale of 1:500, it is clear that in this case the three configurations DG and GeoTag perform better than the standard one. Configuration $\mathrm{B}, \mathrm{C}$, and $\mathrm{D}$ present almost identical values for the mean RMSe on CPs and significantly better than configuration A, especially for the planimetric component. This is particularly interesting for configuration B (DG) where no control points were used as GCPs but all the available control points were used as CPs. The results for B, C, and $\mathrm{D}$ configurations confirm once again the overall good performances of the Phantom 4 RTK adopting an NRTK solution and highlights that not using GCPs will not have a meaningful impact on the positional accuracy. It has to be highlighted that the UAV flight planning took into account the best practices that emerged from previous research work, especially the acquisition of oblique images to make the I.O. (Interior Orientation) and E.O. (Exterior Orientation) parameters estimation more robust. This is one of the reasons of 
the overall good accuracies of the DG and Geotag approaches despite the complex orography of the area (that present and elevation change from $945 \mathrm{~m}$ AMSL to $1100 \mathrm{~m}$ AMSL in around 2 kilometers).

It is also interesting to notice how approach A, the standard one without any geolocation information of camera positions, is the one with the higher RMSe on CPs among the four configurations. This confirms the impact that accurate a-priori knowledge of camera positions have on the overall photogrammetric processing, even when accurate GCPs are available. A second analysis focused on the accuracy of camera coordinates estimations adopting different GCP configurations. For this analysis it was decided to use the configuration D as ground reference that was considered the most balanced in terms of distribution and numerosity of both GCPs and CPs. The deviation between the 3D coordinates of cameras position estimated with the different approaches with respect to configuration D was thus computed and reported in Table 5 .

\begin{tabular}{|l|l|c|c|c|}
\cline { 3 - 5 } \multicolumn{2}{c|}{} & $\Delta \mathrm{X}(\mathrm{m})$ & $\Delta \mathrm{Y}(\mathrm{m})$ & $\Delta \mathrm{Z}(\mathrm{m})$ \\
\hline \multirow{2}{*}{ D-B } & Mean & 0 & 0 & -0.002 \\
\cline { 2 - 5 } & St.Dev & 0 & 0 & 0.003 \\
\hline \multirow{2}{*}{ D-C } & Mean & 0 & 0 & -0.001 \\
\cline { 2 - 5 } & St.Dev & 0 & 0 & 0.002 \\
\hline \multirow{2}{*}{ D-A } & Mean & 0.019 & 0.007 & 0.032 \\
\cline { 2 - 5 } & St.Dev & 0.042 & 0.037 & 0.035 \\
\hline
\end{tabular}

Table 5. Deviations between 3D camera coordinates estimated with GCP configurations $\mathrm{A}, \mathrm{B}$ and $\mathrm{C}$ with respect to configuration $\mathrm{D}$ (used as ground reference)

The values reported in

Table 5 confirm what was already underlined for the RMSe of GCPs/CPs in Table 4: the overall good performance of the approaches $\mathrm{B}$ and $\mathrm{C}$ (differences between coordinates of camera position estimated with the different approaches are negligible for the planimetric component and in the range of few millimeters for the height) and the worst performances of the set processed following the standard approach.

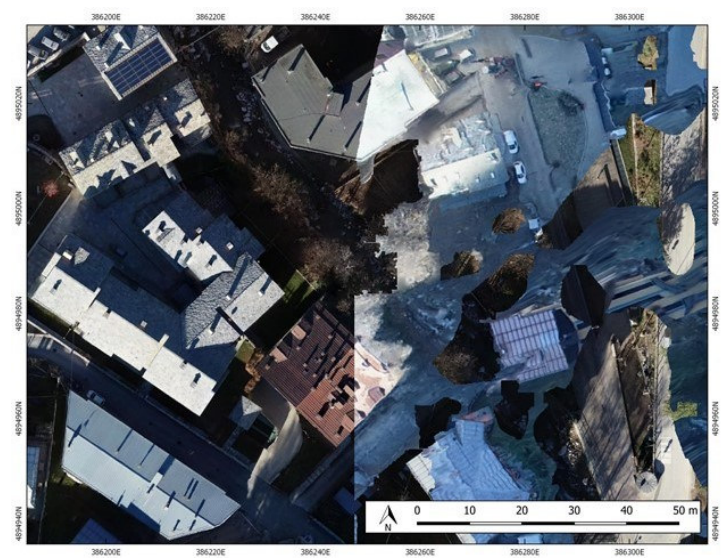

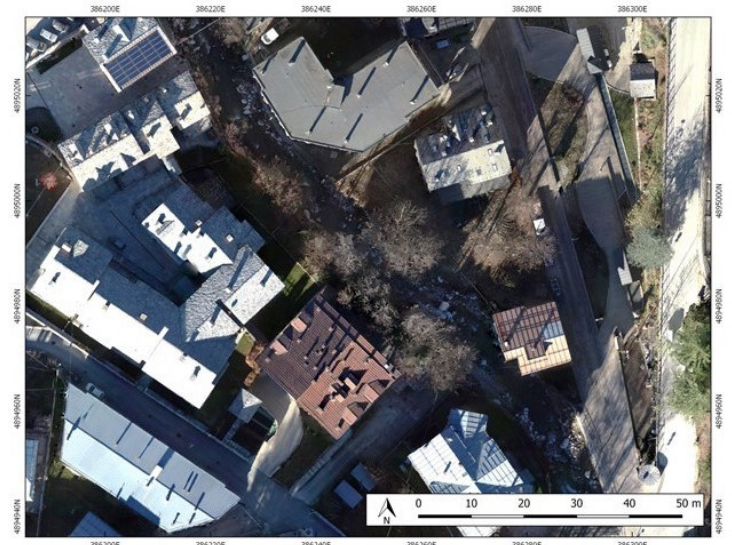

Figure 9. Qualitative comparison between the orthoimages (1:500 map scale) generated adopting Strategy 1 (above) and Strategy 2 (below)

Furthermore, the two different strategies adopted had a meaningful impact also on the overall quality of the generated metric products, such as the orthoimage of the area of Limone Piemonte. In Figure 9 it is clear that the processing of the dataset into separated blocks led to some coverage issues in the overlapping area as well as to an evident lack of radiometric harmonization. On the contrary, the orthoimage derived from Strategy 2 did not presents the aforementioned issue.

\subsection{Camera calibration and Interior Orientation}

Another issue that was analysed, in this case only in the configuration A, B, C, and D of Strategy 2, is the estimation of I.O. parameters. This issue is well described in other works (e.g. Forlani et al., 2018; Gabrlik et al., 2018; Teppati Losè et al., 2020b): the absence, or a low number, of GCPs can results in a wrong estimation of camera I.O. parameters, leading to lower accuracies of the overall photogrammetric processing.

Several strategies can be adopted to deal with this issue as the use of additional GCPs or the availability of a calibration certificate. The DJI Phantom 4 RTK is actually provided with a camera calibration certificate, whose parameters are directly stored in the EXIF file (each image includes focal length, principal point, $\mathrm{k} 1, \mathrm{k} 2, \mathrm{k} 3, \mathrm{p} 1$, and $\mathrm{p} 2$ ). However, as reported in previous research work (Teppati Losè et al., 2020a, 2020b), the data provided by the company are not accurate enough to be used in the photogrammetric processing of a dataset using a precalibration approach for the solution of I.O.

A self-calibration approach was thus adopted for the other configurations to evaluate how they differ from the precalibration certificate and between them. The I.O. parameters derived from the different calibration approaches are reported in Table 6 .

\begin{tabular}{|c|c|c|c|c|c|}
\cline { 2 - 6 } \multicolumn{1}{c|}{} & $\begin{array}{c}\text { F } \\
\text { (pixel) }\end{array}$ & k1 & k2 & k3 & p1 \\
\hline PC & 3635.190 & -0.2635 & 0.1112 & -0.0382 & -0.0009 \\
\hline A & 3629.372 & -0.2681 & 0.1161 & -0.0454 & -0.0007 \\
\hline B & 3630.019 & -0.2682 & 0.1161 & -0.0453 & -0.0007 \\
\hline C & 3630.031 & -0.2682 & 0.1161 & -0.0453 & -0.0007 \\
\hline D & 3630.058 & -0.2682 & 0.1161 & -0.0453 & -0.0007 \\
\hline & $\mathbf{p 2}$ & cx pixel & cy pixel & b1 & b2 \\
\hline PC & -0.0001 & 2.480 & 9.690 & 0 & 0 \\
\hline A & -0.0002 & -3.205 & 5.148 & -0.1197 & 0.0645 \\
\hline B & -0.0002 & -2.486 & 5.102 & -0.1991 & 0.2078 \\
\hline C & -0.0002 & -2.489 & 5.095 & -0.1964 & 0.2079 \\
\hline D & -0.0002 & -2.488 & 5.084 & -0.1949 & 0.2081 \\
\hline
\end{tabular}


Table 6. I.O. parameters provided by DJI (Pre-calibration - PC) and estimated through a self-calibration approach in the four configurations of Strategy 2 (A, B, C, D)

The results of this analysis overlap with the ones of the analyses performed on the GCPs/CPs RMSe (Table 4) and the cameras position (

Table 5). The parameters estimated for the three approaches B, $\mathrm{C}$, and D (DG and Geotag) are almost identical, while the approach A leads slightly different values. These differences are particularly significant for the focal length and the principal point and are one of the elements influencing the overall accuracy of the photogrammetric processing for approach A. Furthermore, the discrepancies of the pre-calibration certificate provided by DJI have been confirmed once again, and its use for photogrammetric processing where the metrical accuracy needs to be controlled and guarantee is not recommended.

\subsection{Satellite data analysis}

As discussed in the previous paragraph (3.1), the satellite based orthoimagery is characterised by a very high spatial resolution $(\mathrm{GSD}=0.5 \mathrm{~m})$ and a $2 \mathrm{D}$ positional RMS lower than $1 \mathrm{~m}$ (validated on $10 \mathrm{CPs}$ ).

Both features (positional accuracy and level of detail) can be also assessed through a qualitative comparison with the UAS orthoimagery, as clearly shown in Figure 10. The images also demonstrate the higher level of detail offered by the UAS acquisitions, that can be extremely relevant to ensure a higher thematic accuracy while assessing damages.

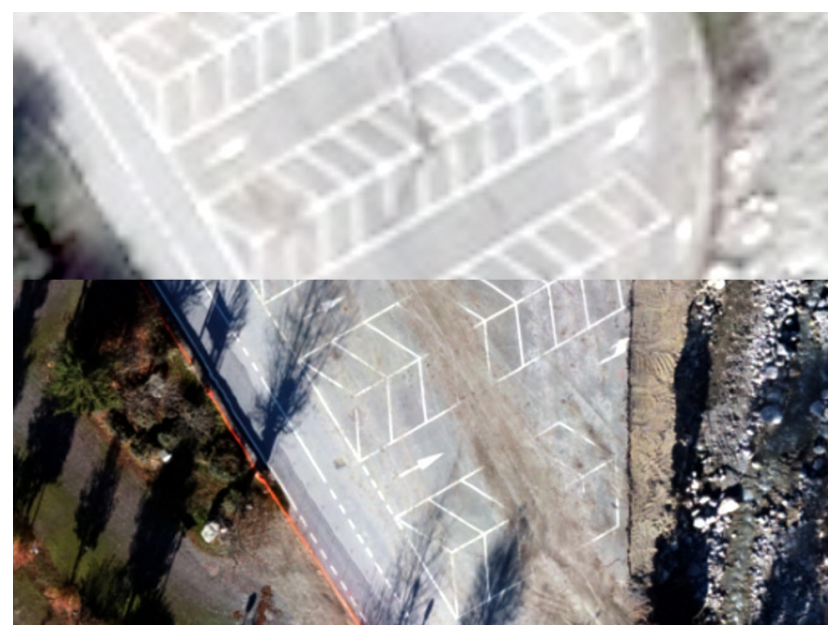

Figure 10. Qualitative evaluation of satellite orthoimagery (top) level of detail and 2D position accuracy with respect to UAS orthoimagery (bottom).

Additionally, a false-colour composite exploiting the NIR band can be used to streamline the visual interpretation and the extraction of thematic information, e.g. vegetation, that appears reddish due to its spectral signature (Figure 11, right). This possibility can be extremely relevant for emergency mapping purpose, enabling the rapid identification of vegetation loss (especially relevant when unsupervised classification algorithms are applied) when compared to pre-event reference dataset. A clear example is shown in Figure 11 (pre-event situation, visible composite, left vs post-event situation, NIR false color composite, right), covering an area affected by a debris-flow leading to vegetation loss.
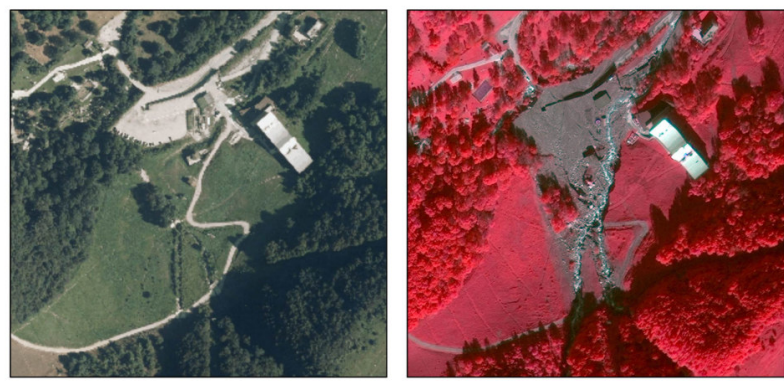

Figure 11. Multi-temporal comparison of a flood-affected area. Reference orthoimagery, visible composite (Regione Piemonte WMTS service, AGEA (C) 2018, left) vs satellite post-event data, NIR false color composite (5 October 2020, right). Vegetation loss due to the flood impact is clearly visible.

The proposed workflow enables the rapid production of postevent orthoimagery as soon as suitable VHR optical satellite data is available.

\section{CONCLUSION AND FURTHER PERSPECTIVES}

The research presented in this manuscript was aimed at analysing different aspects connected both to the photogrammetric acquisition and processing of UAV data and their integration with VHR satellite imagery, both processed following consolidated photogrammetric approaches.

Concerning the UAV dataset the main goal was testing and validating a DG approach thanks to the use of a RTK-enabled UAV. This aspect was crucial especially considering the dimension of the area to be covered by the UAV acquisitions and the effort required for the processing of the ancillary dataset acquired during the survey campaign in the field.

Five different strategies were tested for the processing, without prior knowledge of the camera positions and with/without using GCPs. The different approaches were validated through a set of control points and adopting different $\mathrm{GCPs} / \mathrm{CPs}$ configurations (varying their number and distribution). The RMSe achieved on the control points was the first indicator considered to validate the positional accuracy of these approaches. Nevertheless, further analyses on cameras position estimates confirmed the results achieved with the RMSe analysis.

Further analyses were also performed to calculate the difference of I.O. parameters using the different approaches and the outcomes are compliant with the other results.

The tests carried out on the UAV dataset covering Limone Piemonte confirmed the possibility of processing a large UAV dataset without the use of GCP thanks to the implementation of a DG approach (and theoretically also without $\mathrm{CP}$, that however are considered crucial by the authors to validate the $3 \mathrm{D}$ positional accuracy of the derived metric products). This approach has specific requirements in terms of UAV platform hardware (that should embedded an RTK GNSS receiver) and image acquisition settings (including oblique ones). If the aforementioned requirements are met, it is possible to drastically reduce both field operations and the subsequent data processing maintaining the expected 3D positional accuracies.

Satellite orhoimagery is effectively used for damage assessment purposes (e.g. the activation of the Copernicus Emergency Management Service (C) 2015 European Union), EMSR468, covering the very same event presented in this manuscript) in the emergency response phase (hours/days after the event). Satellite-based products can be synergistically integrated with UAS data - that are generally available only a few days/weeks later (unless a UAS operational service is already set-up before 
the event) - especially in the recovery phase, enabling a multisensor, multi-platform and multi-scale approach.

The UAV 3D models (both point cloud or triangulated mesh) are one of the main advantages with respect to a satellite-based mapping product, since they support first responders in better understanding the real situation in the affected areas (particularly relevant if a pre-event DSM is also available).

Elevation data are also an important asset in the recovery phase, where it can be effectively exploited to derive altimetric profiles, e.g. required for most of the design and planning activities connected to the recovery and reconstruction phase.

\section{ACKNOWLEDGEMENTS}

Authors would like to thank all the participants to the survey campaigns in the area of Limone Piemonte, namely: Berkay Ozgen, Melis Ozalp, Ilaria Cofano as students of the DiRECT team and Prof. Nannina Spanò, Alessandra Spreafico, Francesca Matrone e Stefano Angeli as tutor of the same team.

\section{REFERENCES}

Agüera-Vega, F., Carvajal-Ramírez, F., \& Martínez-Carricondo, P. (2017). Accuracy of digital surface models and orthophotos derived from unmanned aerial vehicle photogrammetry. Journal of Surveying Engineering, 143(2), 1-10. https://doi.org/10.1061/(ASCE)SU.1943-5428.0000206

Aguilar, M. A., Aguilar, F. J., Saldaña, M. del M., \& Fernández, I. (2012). Geopositioning accuracy assessment of GeoEye-1 panchromatic and multispectral imagery. Photogrammetric Engineering and Remote Sensing, 78(3), 247-257. https://doi.org/10.14358/PERS.78.3.247

Boccardo, P., \& Giulio Tonolo, F. (2015). Remote Sensing Role in Emergency Mapping for Disaster Response. In Engineering Geology for Society and Territory - Volume 5 (pp. 17-24). Springer International Publishing. https://doi.org/10.1007/9783-319-09048-1_3

Bolkas, D. (2019). Assessment of GCP Number and Separation Distance for Small UAS Surveys with and without GNSS-PPK Positioning. Journal of Surveying Engineering, 145(3), 1-17. https://doi.org/10.1061/(ASCE)SU.1943-5428.0000283

Calantropio, AlessioCalantropio, A., Chiabrando, F., Sammartano, G., Spanò, A. T., \& Teppati Losè, L. (2018). UAV strategies validation and remote sensing data for damage assessment in post-disaster scenarios. International Archives of the Photogrammetry, Remote Sensing and Spatial Information Sciences - ISPRS Archives, 42(3W4). https://doi.org/10.5194/isprs-archives-XLII-3-W4-121-2018

Chiabrando, F., Giulio Tonolo, F., \& Lingua, A. (2019). Uav direct georeferencing approach in an emergency mapping context. the 2016 central Italy earthquake case study. International Archives of the Photogrammetry, Remote Sensing and Spatial Information Sciences - ISPRS Archives, 42(2/W13), 247-253. https://doi.org/10.5194/isprs-archives-XLII-2-W13247-2019

Duarte, D., Nex, F., Kerle, N., \& Vosselman, G. (2017). Towards a more efficient detection of earthquake induced façade damages using oblique UAV imagery. International
Archives of the Photogrammetry, Remote Sensing and Spatial Information Sciences - ISPRS Archives, 42(2W6), 93-100. https://doi.org/10.5194/isprs-archives-XLII-2-W6-93-2017

Forlani, G., Asta, E. D., Diotri, F., Morra, U., Id, R. R., \& Santise, M. (2018). Quality Assessment of DSMs Produced from UAV Flights Georeferenced with On-Board RTK Positioning. https://oi.org/10.3390/rs10020311

Gabrlik, P., Cour-Harbo, A. la, Kalvodova, P., Zalud, L., \& Janata, P. (2018). Calibration and accuracy assessment in a direct georeferencing system for UAS photogrammetry. International Journal of Remote Sensing, 39(15-16), 49314959. https://doi.org/10.1080/01431161.2018.1434331

Peppa, M. V, Hall, J., Goodyear, J., \& Mills, J. P. (2019). Photogrammetric assessment and comparison of dji phantom 4 pro and phantom 4 rtk small unmanned aircraft systems. International Archives of the Photogrammetry, Remote Sensing and Spatial Information Sciences - ISPRS Archives, 42(2/W13), 503-509. https://doi.org/10.5194/isprs-archives-XLII-2-W13503-2019

Štroner, M., Urban, R., Seidl, J., Reindl, T., \& Brouček, J. (2021). Photogrammetry Using UAV-Mounted GNSS RTK: Georeferencing Strategies without GCPs. Remote Sensing, 13(7), 1336. https://doi.org/10.3390/rs13071336

Taddia, Y., Stecchi, F., \& Pellegrinelli, A. (2020). Coastal Mapping using DJI Phantom 4 RTK in Post-Processing Kinematic Mode. Drones, 4(2), 9. https://doi.org/10.3390/drones4020009

Teppati Losè, L., Chiabrando, F., \& Giulio Tonolo, F. (2020a). Are Measured Ground Control Points Still Required in Uav Based Large Scale Mapping? Assessing the Positional Accuracy of an Rtk Multi-Rotor Platform. ISPRS - International Archives of the Photogrammetry, Remote Sensing and Spatial Information Sciences, XLIII-B1-2, 507-514. https://doi.org/10.5194/isprs-archives-xliii-b1-2020-507-2020

Teppati Losè, L., Chiabrando, F., \& Giulio Tonolo, F. (2020b). Boosting the timeliness of UAV large scale mapping. Direct georeferencing approaches: Operational strategies and best practices. ISPRS International Journal of Geo-Information, 9(10), 578. https://doi.org/10.3390/ijgi9100578

Tonkin, T. N., \& Midgley, N. G. (2016). Ground-control networks for image based surface reconstruction: An investigation of optimum survey designs using UAV derived imagery and structure-from-motion photogrammetry. Remote Sensing, 8(9), 16-19. https://doi.org/10.3390/rs8090786

Tralli, D. M., Blom, R. G., Zlotnicki, V., Donnellan, A., \& Evans, D. L. (2005). Satellite remote sensing of earthquake, volcano, flood, landslide and coastal inundation hazards. ISPRS Journal of Photogrammetry and Remote Sensing, 59(4), 185198. https://doi.org/10.1016/j.isprsjprs.2005.02.002 